\title{
Chronic periaortitis presenting as common bile duct obstruction
}

\author{
D Remedios, M Coppen, J Bradbeer, A Theodossi
}

\begin{abstract}
The case of a 67 year old woman is reported who presented with cholestatic jaundice and was found to have, in addition, an inflammatory abdominal aortic aneurysm. Only at necropsy did histopathology show chronic periaortitis as the aetiology of a pancreatic head mass which, during life, mimicked a pancreatic neoplasm obstructing the bile and pancreatic ducts.
\end{abstract}

The association of chronic periaortitis (perianeurysmal retroperitoneal fibrosis) with duodenal, pleural, and lung fibrosis,' chronic coronary periarteritis, ${ }^{2}$ and ureteric entrapment ${ }^{3}$ have previously been documented. Although there have been reports of common bile duct compression by an abdominal aortic aneurysm ${ }^{4}$ and retroperitoneal fibrosis simulating carcinoma of the pancreas in an anicteric patient, ${ }^{5}$ we believe that this is the first report of a case of chronic periaortitis presenting with cholestatic jaundice and obstruction of the common bile duct.

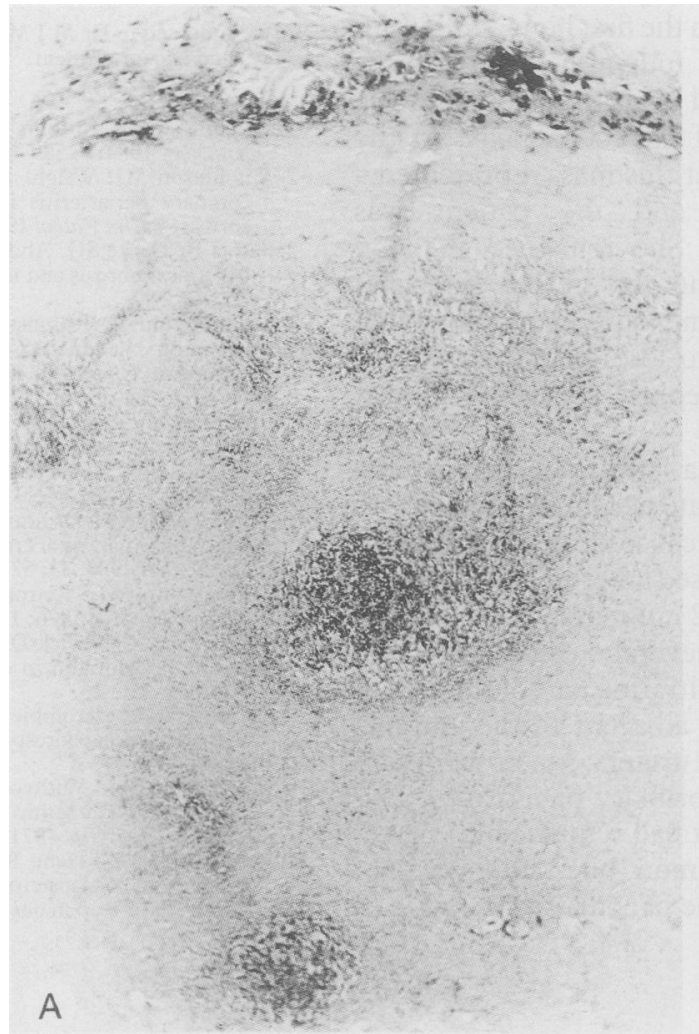

Figure 1: (A) Section of the resected aneurysmal wall showing chronic periaortitis; $(B)$ high power view of fibrotic adventitia (haematoxylin $\mathcal{E}$ eosin).

\section{Case report}

A 67 year old woman was referred with jaundice, vomiting, anorexia, weight loss, and upper abdominal and back pain. She was also found to have a pulsatile mass in the epigastrium.

Her erythrocyte sedimentation rate was $47 \mathrm{~mm}$ in the first hour and 'liver function tests' were abnormal with a serum bilirubin concentration of $479 \mu \mathrm{mol} / \mathrm{l}$ (normal range 10-20), an alkaline phosphatase activity of $2230 \mathrm{IU} / 1$ (normal range 80-300), and transaminases raised fivefold. The ultrasound scan of her abdomen showed intraand extrahepatic duct dilatation but no evidence of a stone or mass in the head of the pancreas. There was also a $4 \mathrm{~cm}$ fusiform abdominal aortic aneurysm.

Endoscopic retrograde cholangiopancreatography (ERCP) showed strictures in the - distal common bile duct and the distal pancreatic duct with prestenotic duct dilatation of both. Using the criteria proposed by Nix et $a l^{b}$ a tumour of the pancreatic head or ampulla of Vater would have been likely. A biliary endoprosthesis was inserted at ERCP to drain the

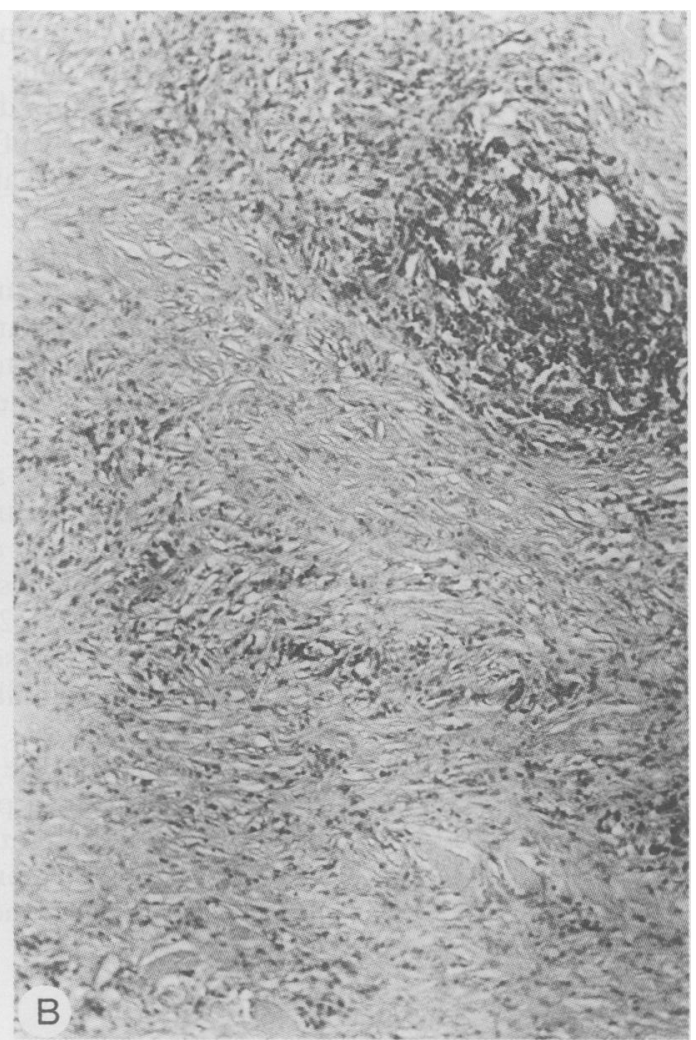

From the Departments of Gastroenterology, Surgery, and Histopathology, Mayday Surrey CR4 7YE

D Remedios

M Coppen

J Bradbeer

Correspondence to:

Dr A Theodossi, Department of Gastroenterology, Mayday Hospital, Croydon, Surre 


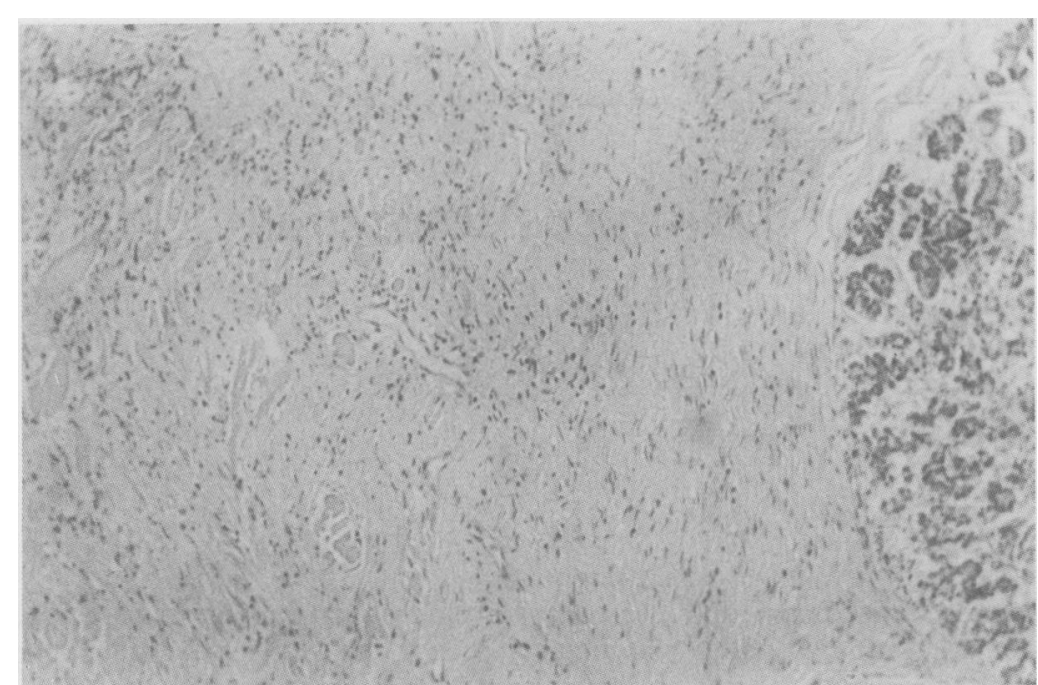

Figure 2: Section from postmortem pancreatic mass showing extensive dense fibrosis (haematoxylin $\mathcal{E}$ eosin). showed extensive dense fibrosis in the peripancreatic tissues adjacent to the site of the previously resected inflammatory aneurysm. Despite careful examination of multiple sections of this pancreatic mass there was no evidence of malignancy. The histological appearances were similar to those in the aneurysmal adventitial tissues.

\section{Discussion}

Chronic periaortitis, as defined, ${ }^{17}$ describes the active chronic inflammation around the lower abdominal aorta accompanied by progressive scarring with venous obliteration and contraction seen, typically, in middle aged and elderly patients, especially men. The erythrocyte sedimentation rate usually exceeds $45 \mathrm{~mm}$ in the first hour. ${ }^{7}$ Parums et al suggested an autoallergic mechanism incriminating the insoluble lipid, ceroid, within atheroma ${ }^{8}$ Corticosteroid treatment has been proferred ${ }^{910}$ and shown by computed tomography in one case ${ }^{11}$ to result in shrinkage of periaortic tissue. Although inflammation may rarely subside spontaneously, it usually does so after treatment with corticosteroids or, curiously, after surgery and even biopsy alone. In this case an endobiliary stent to bypass the stenosing lesion combined with an aneurysectomy resulted in a moderate fall in the erythrocyte sedimentation rate from 47 to $25 \mathrm{~mm}$ in the first hour.

Clearly, periaortitis affecting the pancreatic head may mimic pancreatic malignancy. A readily available serological diagnostic test, possibly involving ceroid, ${ }^{7}$ would prove useful. In the absence of controlled trials we can only speculate if corticosteroids may have helped in this case.

We are indebted to Dr M J Mitchinson for his helpful comments, advice, and encouragement.

1 Mitchinson MJ. Chronic periaortitis and periarteritis. Histo pathology 1984; 8: 589-600

2 Mitchinson MJ, Wright DG, Arno J, Milstein BB. Chronic coronary periarteritis in two patients with chronic periaortitis. 7 Clin Pathol 1984; 37 : 32

3 Sethia B, Darke SG. Abdominal aortic aneurysm with retroperitoneal fibrosis and ureteric entrapment. BrF Surg 1983; 70: 434-6.

4 Van Gossum A, Rubinstein M, Engelholm L, Cremer $M$ Common bile duct compression by an abdominal aortic aneurysm. Endoscopy 1986; 18: 69-70.

5 Perry JF, La Fave JW, Carpenter WB. Retroperitoneal fibrosis simulating carcinoma of the pancreas. Report of a surgically treated case complicated by fatal postoperative hemorrhage. Minn Med 1965; 48: 371-4.

6 Nix GAJJ, Van Overbeeke IC, Wilson JHP, Ten Kate FJW ERCP diagnosis of tumors in the region of the head of the pancreas. Analysis of criteria and computer-aided diagnosis. Dig Dis Sci 1988; 33: 577-86.

7 Mitchinson, MJ. Retroperitoneal fibrosis revisited. Arch Pathol Lab Med 1986; 110: 784-6.

8 Parums DV, Chadwick DR, Mitchinson MJ. The localization of immunoglobulin in chronic pancreatitis. Histopathology of immunoglobuli

9 Clyne CAC, Abercrombie GF. Perianeurysmal retroperitonea fibrosis: two cases responding to steroids. Br f Urol 1977; fibrosis: two cases responding to steroids. $\mathrm{Br} \mathcal{F}$ Urol 1977;

10 Mitchinson MJ, Withycombe JFR, Arden Jones R. The response of idiopathic retroperitoneal fibrosis to corticosteroids. Br F Urol 1971; 43: 444-9.

11 Feldberg MAM, Hene RJ. Perianeurysmal fibrosis and its response to corticosteroid treatment: a computerized tomography follow-up in one case. $\mathcal{F}$ Urol 1983; 130: 1163.

this appeared to be a primary pancreatic car noma. The biliary stent had remained in correct position and the common bile duct was not dilated. Histology of the pancreatic mass (Fig 2) 